\title{
Essais
}

Revue interdisciplinaire d'Humanités

$1 \mid 2012$

Varia

\section{La philosophie comme contre-culture}

\section{Guillaume Le Blanc}

\section{(2) OpenEdition}

Journals

Édition électronique

URL : https://journals.openedition.org/essais/10625

ISSN : 2276-0970

Éditeur

École doctorale Montaigne Humanités

Édition imprimée

Date de publication : 15 mai 2012

Pagination : 7-16

ISSN : 2417-4211

Référence électronique

Guillaume Le Blanc, «La philosophie comme contre-culture », Essais [En ligne], 1 | 2012, mis en ligne le 31 janvier 2022, consulté le 02 février 2022. URL : http://journals.openedition.org/essais/10625 


\section{La philosophie comme contre-culture}

Je vous remercie d'être venus aujourd'hui. Je ne vais pas retracer mon parcours de recherche mais je vais un peu entrer dans le détail de ce que je fais en philosophie depuis maintenant un certain nombre d'années. Naturellement il s'agira là d'une fiction. Mais au fond, l'occasion est belle de se demander ce que je fais avec la pensée, avec la philosophie, avec le travail intellectuel. Il me semble que l'on peut entrer dans le vif du sujet en s'inspirant de ce que Michel Foucault établit dans le cours au collège de France de 1970-1971 qui vient de paraître, Leçons sur la volonté de savoir. Dans la leçon du 9 décembre 1970 qui fait suite à la leçon inaugurale d'entrée au collège de France publiée sous le titre, L'ordre du discours, Foucault propose de résumer ces analyses passées et à venir sous le titre comme ça un peu programmatique de "Fragments pour une morphologie de la volonté de savoir ${ }^{1}$. De tels fragments entendent questionner une histoire des systèmes de pensée (c'est le titre de la chaire que Foucault occupait au Collège de France) depuis la contrainte d'un certain nombre d'opérateurs. Par opérateur Foucault veut indiquer un ensemble d'éléments intérieurs à un type de discours qui permettent de l'identifier et même de le produire comme un discours d'un certain genre. Il distingue en particulier deux opérateurs, les opérateurs philosophiques par lesquels le discours philosophique peut se définir comme tel et plus encore peut se justifier et répondre qu'il est bien un discours philosophique ; les opérateurs épistémologiques qui garantissent la scientificité du discours. Ces opérateurs philosophiques et épistémologiques ne sont nullement extérieurs aux discours qu'ils contribuent à identifier, ce sont des signes que l'on trouve à l'intérieur de ces discours et qui permettent en un sens de les identifier. Tout discours est ainsi ramené à un ordre intérieur qui le définit en propre et qui fait qu’il est un discours philosophique plutôt que littéraire, etc. Ce sont en quelque sorte des traces de certification déposées à l'intérieur des discours par les discours eux-mêmes.

Alors on pourrait dire ceci : s'il y a des ordres de discours qui ne renvoient pas seulement aux conditions historiques et sociales de leur production mais à

1. Michel Foucault, Leçons sur la volonté de savoir, Paris, Gallimard/Le Seuil, 2011, p. 3. 
des signes distinctifs, ces fameux opérateurs, est-ce qu'on ne pourrait pas faire une histoire de ces opérateurs et voir ce qu'ils retiennent, ce qu'ils excluent aussi et ainsi comprendre comment se développe la loi d'une discipline par la production d'un dedans séparé d'un dehors. Chaque fois en effet que des opérateurs viennent dire, ceci est de la philosophie, ceci est de la littérature, ceci est du droit, il faut entendre l'opérateur et le discours qu'il authentifie avec lui comme faisant violence à l'égard d'un ensemble de signes qui sont reconnus comme ne cadrant pas avec la grille d'intelligibilité des opérateurs. De telle sorte qu'à chaque fois qu'il y a quelque chose qui s'affirme comme un certain savoir, il y a autre chose qui est rejeté, qui n'est pas retenu. Le jeu d'une discipline suppose du hors-jeu mais il serait faux de croire que ce hors-jeu est extérieur au jeu. En réalité, il est ce non-savoir qui est précisément expulsé par le jeu de la discipline pour se comprendre comme savoir. Ainsi Foucault, dans la même leçon, montre que, la philosophie dès lors qu'elle se définit avec Aristote comme un savoir par nature, exclut le savoir tragique comme un savoir qui, justement, n'est pas désiré par nature, comme un savoir qui inquiète le type de savoir qu'il est convenu de désirer par nature. Et la question devient alors la suivante, et je dirais que c'est cette question qui est finalement la mienne : comment penser le hors-jeu du savoir constitué, comment penser pour la philosophie quelque chose comme son dehors? Il s'agit en somme de s'intéresser aux poubelles des disciplines, à ce qui n'est pas retenu comme de la connaissance mais qui ne fonctionne pas seulement comme un rebus qu'il faut situer à l'extérieur mais aussi comme un spectre qui fait retour dans la discipline elle-même. Il me semble que faire les poubelles de sa propre discipline peut être intéressant et définir une certaine attitude que je nomme en philosophie la contre-culture. Je ne dirais pas que mon travail s'est toujours défini ainsi mais je dirais qu'il a pris en revanche cette orientation. En ce sens, faire de la philosophie c'est faire une critique de ce qui est présupposé comme étant de la philosophie et c'est ce que j'essaie de faire depuis une orientation générale consistant à soutenir que la philosophie n'existe que par référence à un dehors qu'elle congédie et que j'essaie, pour ma part, avec d'autres, de faire rentrer dans l'analyse de la philosophie.

\section{La philosophie et son dehors}

Ce n'est pas un hasard, je crois, si mes premiers travaux ont porté sur Canguilhem. N'est-ce pas Canguilhem qui dès la préface de son livre-princeps, Le normal et le pathologique, publié sous sa version définitive en 1966, affirmait que « la philosophie est une réflexion pour qui toute matière étrangère est bonne et nous dirions volontiers pour qui toute bonne matière doit être étrangère $n^{2}$.

2. Georges Canguilhem, Le normal et le pathologique, Paris, PUF, 1966, p. 7. 
Cette référence à la "matière étrangère ", en l'occurrence pour Canguilhem, l'étude de la médecine, signale que le "dedans " de la philosophie ne vaut que par référence à un " dehors ». Faire entrer ce "dehors » dans ce "dedans » pour le réfléchir, c'est, je crois, abîmer ce dedans présumé, par l'appel à une série de problème que ce "dedans " ne se pose pas ou ne veut pas se poser. En particulier pour Canguilhem dont je rappelle qu'il fut le directeur de thèse de Michel Foucault qui soutint L'histoire de la folie sous sa direction, il s'est agi par là même de faire entrer une série de "problèmes concrets " comme la variété des comportements pathologiques mais aussi les rapports entre normes et techniques qui ne peuvent manquer de mener la philosophie vers d'autres directions. Cette irruption du concret est à mettre au service de l'irruption d'un dehors et cette intrusion du dehors est, je crois, la grande affaire de la philosophie française des sixties que Merleau-Ponty résuma en un sens lorsqu'il intitula l'un de ses textes en 1960 "La philosophie et le dehors ». On peut la penser de différentes façons : 1/comme une matière étrangère qui réoriente de fond en comble les pratiques de la philosophie et l'oblige à revenir sur des problèmes en apparence résolus, comme Canguilhem le note lui-même en soulignant avec humour que "la philosophie est la science des problèmes résolus $»^{3}$, voulant dire par là qu'elle a pour rôle de " rouvrir les problèmes plutôt que de les clore $»^{4}$ et que c'est cela faire la science des problèmes résolus. 2/Ce dehors peut être pensé également à partir des limites qui définissent notre culture. Le dehors comme limite. Anticipant le partage qui s'établit à l'âge classique entre raison et folie, Foucault note, dans sa première préface, que l'" on pourrait faire une histoire des limites, de ces gestes obscurs, nécessairement oubliés dès qu'accomplis, par lesquels une culture rejette quelque chose qui sera pour elle l'Extérieur ${ }^{5}$. Ici, le dehors ne vaut pas comme ce qui vient redéfinir le dedans mais comme ce qui est rejeté par le dedans. Ce rejet se signale dans le geste de tracer une limite entre le dedans et le dehors à la manière de la séparation produite par l'âge classique entre raison et folie, illustrée à titre d'exemple par la manière dont Descartes prend congés de la folie sous la forme d'une sentence irrévocable: "Mais quoi ce sont des fous et je ne serais pas moins extravagant, si je me réglais sur leurs exemples ${ }^{6}$, commentée vivement par Foucault. 3/Le dehors peut être pensé également comme une marge à la manière de Derrida, c'est-à-dire comme un ensemble de positions qui tiennent dans le texte lui-même mais à titre d'écarts qui assurent la ligne de flottaison de

3. Ibid., p. 9.

4. Ibid.

5. Michel Foucault, "Préface" à Folie et déraison. Histoire de la folie à l'âge classique in Frédéric Gros et Arnold Davidson (sous la dir. de), Michel Foucault anthologie, Paris, Gallimard, 2004, p. 52.

6. Descartes, Méditations métaphysiques, Paris, Vrin, 1996, Euvres complètes, t. IX, p. 14. 
la norme textuelle et en même temps la déstabilisent. On sait que la question de Derrida était bien la suivante : "Comment penser le dehors d'un texte ? Plus ou moins que sa propre marge ? $»^{7}$. Car, pour Derrida, si la philosophie n’a eu de cesse de régler les différences, de régler la polysémie par la référence à une unité cachée qui organise par avance le divers, "surveillant ses marges ${ }^{8}$, l'appel à un dehors, sous la forme d'une référence non capturée à la marge est alors ce qui doit permettre au texte philosophique de différer d'avec lui-même sous forme de traces qui ne se laissent pas capturer par l'unité présumée d'une signification primordiale. 4/Enfin le dehors peut être l'autre discours qui ne se laisse pas cadrer par l'ordre des discours que nous étudions principalement : la littérature chez Deleuze par rapport à la philosophie, les sciences humaines pour Merleau-Ponty par rapport à la philosophie. Alors il faut dire que chacun de ces dehors vient contester ce qui présente comme dedans. La référence à une matière étrangère défait l'ordonnancement d'une philosophie première. De même, la référence aux limites de notre culture vient faire apparaître les types de violence et d'exclusion qui fabriquent notre culture. Également, la marge vient faire différer le texte de lui-même, le rendre différand. Enfin, les pensées produites par les autres discours exorbitent la philosophie, la décentrent d'ellemême.

Alors je dirais comme ça puisqu'il faut bien se lâcher que faire entrer le dehors dans la philosophie c'est ce que j'essaie de faire, de plusieurs manières, en menaçant le type de rationalité philosophique par d'autres rationalités, empruntées à d'autres disciplines comme la médecine mais aussi les sciences sociales d'une part ; en revenant d'autre part sur les lignes de partage entre le dedans présumé de la philosophie et son dehors. Ce que j'essaie de faire aujourd'hui avec l'idée d'une archéologie de la bioéthique me semble relever de cet état de fait. Si la philosophie a pu se redonner une modalité éthique particulièrement importante sous l'appellation de bioéthique, qu'elle soit d'inspiration kantienne ou utilitariste, de facture normative ou conséquentialiste, plutôt que d'analyser les différents types de légitimation philosophique de la bioéthique, il me semble plus important d'analyser ses conditions d'apparition historique comme pratique philosophique mais aussi comme pratique sociale cherchant à s'emparer d'une matière comme la médecine pour la réfléchir selon la constitution d'un droit sans valeur juridique. S’intéresser aux conditions historiques d'apparition de la bioéthique, c'est faire apparaître deux actes de la naissance de la bioéthique qui excèdent la légitimité philosophique, puisque le premier se situe dans la perspective d'une écologie fondamentale tandis que le second, seulement, se situe dans la perspective

7. Jacques Derrida, Marges de la philosophie, Paris, Éditions de Minuit, 1972, p. 27.

8. Ibid., p. XXIV. 
d'une critique de la raison médicale. Comprendre pourquoi la seconde a pris le dessus sur la première, c'est alors retracer un certain nombre d'arguments sur le consentement, sur le don (d'organes, de sang, de gamètes), sur l'injonction à la narration sans soupçonner qu'il existe un "universel " de la bioéthique et en étant plutôt attentif aux tactiques de pouvoir et aux jeux de savoir par lesquels l'universel présumé de la bioéthique vient unifier de façon fallacieuse une matière étrangère. Ici encore, je me référerais volontiers à Foucault et à sa décision théorique de supposer l'inexistence des universels pour pouvoir en faire l'histoire. Dans la leçon du 10 janvier 1979 qui ouvre son cours au collège de France intitulé Naissance de la biopolitique, Foucault distingue sa méthode philosophique de l'historicisme. Ce dernier présuppose l'existence de l'universel et se propose d'en faire l'histoire, il fait de l'histoire une méthode critique d'interrogation des universaux tandis que la décision théorique de Foucault est de suspendre la thèse de l'existence de l'universel afin de faire apparaître les événements et les pratiques qui s'ordonnent en apparence à un universel, par exemple celui de la folie?

Il me semble que mon intérêt pour les sciences sociales est de même nature. Il s'agit bien de déplacer le centre de gravité de la philosophie au-dehors d'elle-même pour souligner précisément ce qu'elle a tendance à retenir ou à occulter et que d'autres discours peuvent contribuer à mettre en avant. Non pas que la vérité de la philosophie se situe dans les autres discours mais que la confrontation entre des ordres de discours qui obéissent à des opérateurs distincts, seule, permet de rendre compte d'une expérience. L'expérience ne désigne pas, dans mon esprit, ce qui est antérieur au savoir et qui ne peut être explicité que par un savoir à venir qui s'en empare. Elle vaut plutôt comme une explicitation interne à des conflits de savoirs. Elle est ce qui s'explicite à l'intérieur de rapports de tension, de conflits mais aussi parfois d'analogie entre les opérateurs qui identifient des ordres de savoirs distincts. Si l'on en reste à la philosophie et même à la philosophie sociale, on aura alors, pour rendre compte de l'expérience sociale, les catégories d'aliénation, de réification, de classe, etc. mais jamais quelque chose comme la précarité par exemple ou l'exclusion. Pour atteindre de telles catégories et ainsi viser une expérience qu'elles manifestent et peuvent occulter aussi, il faut bien alors s'emparer de discours sociologiques, juridiques, politiques qui rendent visible quelque chose comme la précarité. Mais ce qui est particulièrement intéressant, c'est le heurt qui en résulte entre le concept de précarité ainsi reconstitué à partir des travaux de Paugam sur la disqualification sociale, de Lapeyronnie sur la ségrégation sociale, de Castel sur la désaffiliation, et les concepts philosophiques dont nous

9. Michel Foucault, Naissance de la biopolitique, Cours au Collège de France 1978-1979, Paris, Gallimard/le Seuil, 2004, Leçon du 10 janvier 1979, p. 5. 
venons de parler. Je crois que c'est en problématisant cette zone de friction entre des ordres de discours différents que quelque chose comme le sens d'une expérience peut être explicité. Attention au terme d'expérience : elle ne désigne plus ici ce qui précède un savoir mais bien ce qui est explicité par la mise en relation et en tension entre des savoirs distincts. Comme le souligne l'historienne américaine Joan W. Scott, «l'expérience devient non pas ce sur quoi s'appuie notre explication, non pas la preuve incontestable qui ancre ce que nous savons ; elle devient plutôt ce que nous cherchons à comprendre, ce sur quoi du savoir sera produit». Tout le contraire donc d'une évidence de l'expérience d'où procéderait le savoir mais la construction d'une expérience à la jonction des savoirs hétérogènes. "L'expérience est à la fois quelque chose qui est déjà une interprétation et quelque chose qui doit être interprété » ${ }^{10}$.

\section{La philosophie comme culture}

Ainsi, le rôle d'une recherche me semble être (en tout cas c'est ainsi que je vois les choses) de questionner les lois de partage, produites dans les discours entre des ordres de discours distincts, dont les uns s'octroient la caractéristique de construire un dedans présumé de ces discours distinct d'un dehors tout autant présumé. Cette interrogation sur les partages dedans/dehors, je me rends compte maintenant seulement que j'ai cherché à la généraliser non seulement dans le mode d'interrogation sur l'ordre des discours qui attachent les uns à la qualité de discours philosophiques par opposition aux autres mais aussi en questionnant les partages sociaux qui assignent les uns à l'espace du dedans et les autres à l'espace du dehors ou de ce que je me suis proposé de nommer dans mon dernier essai, Dedans, dehors. La condition d'étranger, un dedans-dehors. Je m'efforce en effet de comprendre selon quelles modalités dans l'ordre des savoirs mais aussi dans l'ordre des pouvoirs certaines vies sont assignées à un espace du dehors depuis un ensemble de déterminations qui fonctionnent comme la loi du dedans, au point qu'elles en viennent à la limite à être représentées comme irreprésentables. J'essaie ainsi de traquer les exclusions des vies hors des espaces sociaux consacrés en m'attachant à définir ce processus d'exclusion comme un processus qui attache aux uns la possibilité de se définir et de se vivre comme "dedans " en débarquant les autres comme "dehors " même s'ils sont aussi parfois « dedans ». Il y a ici une complémentarité forte entre le fait de se définir comme le groupe des "uns» en débarquant des vies et en leur attachant la qualité d'être des vies autres et de s'octroyer la caractéristique sociale d'être dedans en refusant cette caractéristique pour d'autres vies. Christine Delphy en a bien souligné les enjeux à la fois épistémologiques et sociologiques dans un essai de 2008 intitulé "Les Uns derrière les Autres » lorsqu'elle a soutenu

10. Joan W. Scott, Théorie critique de l'histoire, Paris, Fayard, 2009 pour la traduction française, p. 125. 
que les Uns sont ceux qui peuvent définir les Autres tandis que «les Autres, justement parce qu'ils sont Autres, ne peuvent appeler les Uns des Autres »" Le partage entre le dedans et le dehors relève à la fois de classements et de gestes d'exclusion, de divisions et de constructions de hiérarchies. L'autre est à la fois celui qui est mis de côté, qui n'est pas retenu dans les classements officiels et qui est débarqué du groupe des uns. C'est pourquoi l'opposition du dedans et du dehors ne renvoie pas qu'à des partages entre les ordres des discours mais elle fonctionne toujours également comme la production pratique d'une ligne de distinction entre les vies qui sont reconnues comme "dedans » et celles qui sont reconnues comme "dedans" et aussi "dehors". En cherchant à analyser le précaire comme un sujet situé à la fois dedans et dehors, inclus et exclus, en construisant une analyse de la figure de l'étranger, comme étant assigné dans le dedans d'une nation à l'espace du dedans-dehors, j'ai voulu souligner combien les procédés d'altérisation de certaines vies fabriquées comme vies autres doivent être compris comme des formes de qualification et de disqualifications produites par différents relais de pouvoir à l'intérieur des univers sociaux consacrés. Cette analyse des procédures sociales, juridiques, politiques de relégation de certaines vies qui en viennent de ce fait à se vivre comme des vies irreprésentables, dans mon esprit, ne doit pas être séparée de l'analyse des différentes formes d'exclusion par lesquelles un genre de discours se garantit comme discours d'un certain genre. En ce qui me concerne, revenir sur les partages pratiques entre un dedans et un dehors, c'est, dans le même temps, questionner le type d'hégémonie culturelle que peut représenter un ordre des discours particulièrement vigoureux comme par exemple le discours philosophique lorsqu'il a voulu se comprendre du côté de l'énonciation de l'universel et qu'il a, de ce fait, réitéré ou même mieux produit des partages entre certaines formes de vies qui ont accès à l'universel et d'autres formes qui n'y ont pas accès. Revenant ainsi sur l'histoire de la philosophie, il s'agit pour moi d'analyser la philosophie à partir de ce qu'elle exclut, qu'elle ne retient pas comme digne d'être représenté, l'ouvrier, la femme, l'enfant, l'étranger, etc., définissant quelque chose comme son Autre pour mieux s'assurer de la possibilité de séjourner dans l'universel. On peut penser aux nombreuses exclusions produites par la philosophie, celle en tout premier plan, analysée par Rancière dans le livre de 1983, Le philosophe et ses pauvres, qui fait dire à Platon que la divinité a donné aux uns l'âme d'or des philosophes et aux autres l'âme de fer des artisans, produisant un partage sans appel entre les philosophes qui s'occupent de la cité et les cordonniers qui ne s'occupent que de leurs chaussures ${ }^{12}$. On peut penser aussi à l'exclusion des femmes hors de la

11. Christine Delphy, Classer, dominer, Paris, la Fabrique, 2008, p. 19.

12. Jacques Rancière, Le philosophe et ses pauvres, Paris, Fayard, 1983. 
cité d'Aristote, à la loi de partage produite par Kant entre les différentes races, à la construction d'une humanité africaine aux limites de l'humanité produite par Hegel dans sa Raison dans l'histoire au nom de l'appel à un universel qui se trouve être un universel fortement européen, à la loi de partage réitérée par Husserl dans sa conférence de Vienne prononcée en 1935 «La crise de l'humanité européenne et la philosophie » entre une humanité européenne qui comprend également les Américains et des habitants qui se voient refuser le titre d'européens, les Eskimos mais aussi les Tsiganes. On se souvient de l'énoncé : «Au sens spirituel, il est manifeste que les dominions anglais, les États-Unis, etc., appartiennent à l'Europe, mais non pas les Esquimaux ou les Indiens des ménageries foraines, ni les Tziganes qui vagabondent perpétuellement en Europe. ${ }^{13}$ L'unité de vie européenne est refusée dans le même geste, dans l'ordre du discours philosophique, aux Esquimaux, aux Indiens et aux Tziganes en même temps que ce refus souligne l'appartenance du discours philosophique à ce qu'il accrédite, à savoir l'unité spirituelle réalisée précisément par la philosophie grecque en son commencement. En réalité, si l'on analyse le type d'exclusion produit ici par la philosophie lorsqu'elle témoigne d'elle-même quand elle témoigne contre les autres, on peut remarquer que la philosophie produit ou amplifie des partages entre l'Europe et l'Afrique (Hegel), entre l'Europe et ses étrangers (Husserl) chaque fois qu'elle prétend parler au nom de l'universel et qu'elle rechigne à se penser comme un discours culturellement contextualisé. Ainsi, c'est chaque fois que la philosophie renonce à penser sa propre particularité culturelle qu'elle en vient à produire un universel qui est en réalité l'expression d'une préférence culturelle non thématisée comme telle. Par contraste, penser l'ordre des discours philosophique comme un ordre culturellement contextualisé qui intervient selon certaines conditions institutionnelles, sociales, politiques, c'est alors se donner la possibilité de nouvelles orientations normatives qui déstabilisent les partages épistémiques entre les ordres de discours, entre les connaissances et les savoirs et aussi les partages sociaux entre les uns et les autres.

\section{La philosophie comme contre-culture}

Je souhaiterais approfondir dans un dernier moment de mon intervention cette compréhension culturaliste de la philosophie et en tirer toutes les conséquences. Deux possibilités s'offrent, dans ce contexte culturaliste, à la philosophie aujourd'hui. 1/On peut opter pour une analyse des jeux de langage propres aux différents types d'énoncés qui ont à voir avec la philosophie, en décrire les modalités propres en fonction des formes de vie auxquelles renvoient ces jeux de langage et ainsi renoncer à toute position universaliste au profit

13. Husserl, La crise des sciences européennes et la phénoménologie transcendantale, Paris, Gallimard, 1976 pour l'ensemble de la traduction française, p. 352. 
d'une position culturaliste. Au paragraphe 23 des Investigations philosophiques de Wittgenstein, on trouve la remarque suivante : " Le mot jeu de langage doit faire ressortir ici que le parler du langage fait partie d'une activité ou d'une forme de vie ». Ceci revient à dire que la tâche de la philosophie n'est plus de proposer un universel abstrait surplombant toutes les situations et risquant, de ce fait, de reconduire des préférences culturelles implicites attachées à la formulation de cet universel, mais de circonscrire des énoncés relativement à des jeux de langage eux-mêmes en rapport à des formes de vie particulières. Il s'agit donc bien d'une orientation culturaliste de la philosophie où la nécessité thérapeutique de soigner des illusions métaphysiques qui reconduisent des universaux surplombants va de pair avec la prise en considération des contextes culturels en rapport avec des formes de vie particulières. Au $\$ 241$ il explicite ce raisonnement de la manière suivante : "Ainsi vous dîtes que la conformité des vies humaines décide de ce qui est vrai et de ce qui est faux ? Est vrai et faux ce que les hommes disent l'être; et ils ne s'accordent pas dans le langage qu'ils emploient. Ce n'est pas une conformité d'opinion mais de forme de vie. "Ce passage signale notamment que la possibilité de dire le vrai et conséquemment le faux ne relève, en dernière instance, d'aucun critère transcendantal mais de la forme de vie à laquelle sont liés ceux qui énoncent un jugement. Il faut donc se retourner vers cette forme de vie pour en comprendre le lien avec le type de jeu de langage qu'elle sous-tend et cette perspective suppose de prendre congés de toute possibilité, exorbitante pour tout dire, de se dégager d'une forme de vie particulière pour énoncer un universel. Selon cette première perspective, la question est celle de la conformité supposée entre un jeu de langage et une forme de vie, c'est-à-dire que la question centrale est celle de savoir si nous sommes fondés eu égard à nos formes de vie à mobiliser tel ou tel jeu de langage. Si l'on accorde un sens culturel étroit à la forme de vie, ce que les études préliminaires suggèrent, en identifiant langage et culture, alors la conséquence est qu'il existe autant de formes de vie que de systèmes culturels et que, de ce fait, il est impossible d'envisager une grammaire qui comparerait ces systèmes culturels ; tout au plus peut-on comprendre le sens pratique de l'usage des règles à l'intérieur d'une forme de vie particulière. Wittgenstein le confirme au $\$ 584$ : «Le couronnement d'un roi est une image de la magnifiscience et de la dignité. Isolez de son ambiance une phase de la cérémonie : on pose la couronne sur la tête du roi revêtu de la tunique du couronnement. Mais voici que dans une autre ambiance l'or est le moins précieux des métaux, son éclat passe pour tout à fait ordinaire. Le tissu de la tunique y est à vil prix. Quant à la couronne elle apparaît comme la parodie d'un respectable chapeau, etc. ${ }^{14}$ Il ressort clairement de cet exemple que la fonction attribuée à la couronne

14. Cité par Fabrice Clément, "Une nouvelle forme de vie pour les sciences sociales » in Revue européenne des sciences sociales, XXXIX, 106. 
et donc aussi au couronnement dépend étroitement du système culturel dans lequel cette fonction est prélevée. Le rôle de la philosophie est alors de s'assurer de la conformité entre un jeu de langage et une forme de vie. 2/Il me semble qu'on peut opter pour une deuxième analyse et c'est sur elle que je voudrais conclure. Tandis que la première analyse de la philosophie comme discours culturellement contextualisé aboutit à la suspension de toute thèse qui ne pense pas son appartenance à un mode culturel particulier, conduisant à une interrogation sur le sens pratique de suivre les règles dans un système culturel particulier et sur la manière de valider ou d'invalider des jeux de langage particulier en rapport à des formes de vie singulières, une seconde analyse, d'inspiration plus foucadienne, sous laquelle je me range, consiste à, partir de cette inscription culturelle de la philosophie, à envisager les modes de critique possibles et féconds, quelque chose donc comme une contre-culture: il ne s'agit pas seulement de décrire d'où l'on parle mais de critiquer certains des sites à partir desquels on est amené à dire ce que l'on dit, déployant donc dans l'entreprise d'une histoire culturelle de nos discours qu'elle soit de facture archéologique ou généalogique comme une critique, au sens que Foucault a donné à ce terme dans sa conférence de 1978 "Qu'est-ce que la critique ? ", à savoir un certain " art de l'inservitude volontaire, celui de l'indocilité réfléchie ", au sens également que la traductrice de la Dissémination de Derrida, Barbara Johnson, donne à ce terme : "La critique d'un système théorique quel qu'il soit n'est pas un examen de ses faiblesses et de ses imperfections. Ce n'est pas une série d'observations critiques destinées à l'améliorer. C'est une analyse qui se concentre en priorité sur les possibilités qu'offre le système. Partant de ce qui paraît naturel, allant de soi ou universel, la critique remonte vers l'amont de ce qu'elle étudie, de manière à montrer que ces choses ont leur histoire, qu'on peut analyser les raisons qui les font être ce qu'elles sont ainsi que les effets qu'elles produisent ; et que le point de départ n'est pas un donné naturel mais une construction culturelle, presque toujours aveugle à son propre sujet. ${ }^{15}$

15. Cité par Joan W. Scott, Théorie critique de l'histoire, op. cit., p. 27-28. 